\title{
Does local implantation of gentamicin impair renal function in patients undergoing surgery for chronic bone infection?
}

\author{
Ross Muir, Catherine Birnie, Robert Hyder-Wilson, Jamie Ferguson, Martin A. McNally*
}

The Oxford Bone Infection Unit, Nuffield Orthopaedic Centre, Oxford University Hospitals, United Kingdom

Received: 05 February 2021

Accepted: 12 March 2021

*Correspondence:

Martin A. McNally,

E-mail: martin.mcnally@ouh.nhs.uk

Copyright: () the author(s), publisher and licensee Medip Academy. This is an open-access article distributed under the terms of the Creative Commons Attribution Non-Commercial License, which permits unrestricted non-commercial use, distribution, and reproduction in any medium, provided the original work is properly cited.

\begin{abstract}
Background: The treatment of chronic bone infection often involves excision of dead bone and implantation of biomaterials which elute antibiotics. Gentamicin is a preferred drug for local delivery, but its systemic use carries a well-established risk of nephrotoxicity. We aim to establish the risk of acute kidney injury (AKI) with local delivery in a ceramic carrier.

Methods: 163 patients with Cierny-Mader type 3 or 4 chronic osteomyelitis had a single-stage operation including filling of the osseous defect with a calcium sulphate-hydroxyapatite carrier containing gentamicin. Mean gentamicin dosing was $191.3 \mathrm{mg}$ (maximum $525 \mathrm{mg}$ ). Glomerular filtration rate (GFR) was calculated pre-operatively and during the first seven days post-operatively. Renal impairment was graded using the chronic kidney disease (CKD) staging system, and AKI was assessed using the RIFLE criteria.

Results: 155 cases had adequate data to allow calculation of pre- and post-operative GFR. 7 had pre-existing renal disease. 70 patients $(45.2 \%)$ had a temporary GFR drop post-operatively, with the greatest decrease occurring at a mean of 3.06 days following surgery. Twenty cases had a $>10 \%$ decline in GFR, but 12 resolved within 7 days. 7 patients transiently fell into the "Risk" category according to RIFLE criteria, but no patient had a change consistent with "Injury", "Failure" or "Loss" of renal function and none had clinical signs of new acute renal impairment postoperatively.

Conclusions: Renal function is not significantly affected by local implantation of gentamicin up to $525 \mathrm{mg}$. The presence of pre-existing renal disease is not a contraindication to local gentamicin therapy.
\end{abstract}

Keywords: Osteomyelitis, Gentamicin, Nephrotoxicity, Surgery, Biomaterial

\section{INTRODUCTION}

Chronic bone infection requires a combined medical and surgical approach for effective treatment. ${ }^{1,2}$ The main treatment principles are adequate excision of infected and devitalised bone and soft tissue, control of dead space, skeletal stabilisation, culture-specific antimicrobial therapy and robust soft tissue coverage..$^{3-5}$

Surgical excision is aimed at removing all dead tissue and the associated biofilm, but it is impossible to eradicate every bacterium and small fragment of biofilm. Antibiotic therapy is therefore essential to treat these remaining bacteria and prevent reinfection. Broadspectrum empiric antibiotics are initially used but these should be changed based on the culture of deep intraoperative microbiological samples as soon as is practical. $^{6}$

Traditional teaching dictates that systemic antibiotics, either intravenous or oral, are typically given for a period of 4-6 weeks following excision of osteomyelitis, but with prolonged systemic exposure, side effects are frequently seen. In the OVIVA trial, where over 1000 patients with bone infections were randomised to either oral or intravenous antibiotics, over $10 \%$ of patients 
suffered serious adverse events related to the antibiotic therapy. ${ }^{7}$ However, the effectiveness of systemic antibiotics post-operatively has been shown in animal studies to be limited by haematoma, hypoperfusion and acidosis at the surgical site leading to limited tissue penetration of the circulating drug. ${ }^{8-10}$ Also, residual bacteria in biofilm can only be killed by very high levels of antimicrobials and it is unlikely that these can be reliably achieved by systemic administration alone. ${ }^{11}$

For these reasons, the insertion of local antibiotics contained within a carrier compound into bone defects at the time of surgery has become increasingly popular. These substances fill the dead space within the bone, and release very high concentrations of antibiotics into the tissues for a number of weeks after the operation. ${ }^{12-15}$ Many antibiotics have been employed for local administration, but aminoglycosides, and gentamicin in particular, are by far the most widely used. Nephrotoxicity is a well-recognised side effect seen with the systemic use of these drugs, even after a single dose and the deleterious effect on renal function is increased with higher doses and longer courses of treatment. ${ }^{16,17}$ Theoretically, local use of aminoglycosides could reduce systemic side-effects. ${ }^{18,19}$ However, modern bioabsorbable antibiotic carriers are designed to release an initial 'burst' of gentamicin with levels many times above the mean biofilm eradication concentration (MBEC). This release reaches its highest levels in the first few days after implantation but will continue, at a reducing level for several weeks. ${ }^{20}$ Most commercially available antibiotic carriers are supplied with a fixed concentration of gentamicin and larger bone defects (requiring large volumes of carrier to fill) will lead to high doses of gentamicin being implanted. For this reason, there may be concern that this places patients at risk of acute kidney injury. This has not previously been investigated with bioabsorbable antibiotic carriers.

This study evaluated the pre- and post-operative renal function of a consecutive series of patients treated for osteomyelitis using a gentamicin-containing ceramic antibiotic carrier (Cerament G, Bonesupport AB, Lund, Sweden) to ascertain the risk and degree of renal injury with this treatment.

\section{METHODS}

163 consecutive patients with Cierny-Mader type III and IV chronic osteomyelitis who had been treated according to a single-stage protocol for chronic osteomyelitis were identified from a departmental database., ${ }^{1,21}$ Data was extracted from medical records and operative notes on demographics, pre-existing medical comorbidities, including renal impairment and surgical findings.

Surgery was performed according to an established protocol, comprising removal of sinuses and metalwork and excision of dead bone, after taking multiple deep samples for microbiology and histology. ${ }^{21}$ The surgical site was lavaged with $0.05 \%$ aqueous chlorhexidine and the resultant bone void was then dried and filled with Cerament G, containing $17.5 \mathrm{mg}$ of gentamicin per millilitre of carrier. No other implanted antibiotic carriers were used. Soft tissue closure was achieved in all cases either with direct primary closure, or using local or free flaps (Figure $1 \mathrm{~A}-\mathrm{C}$ ).
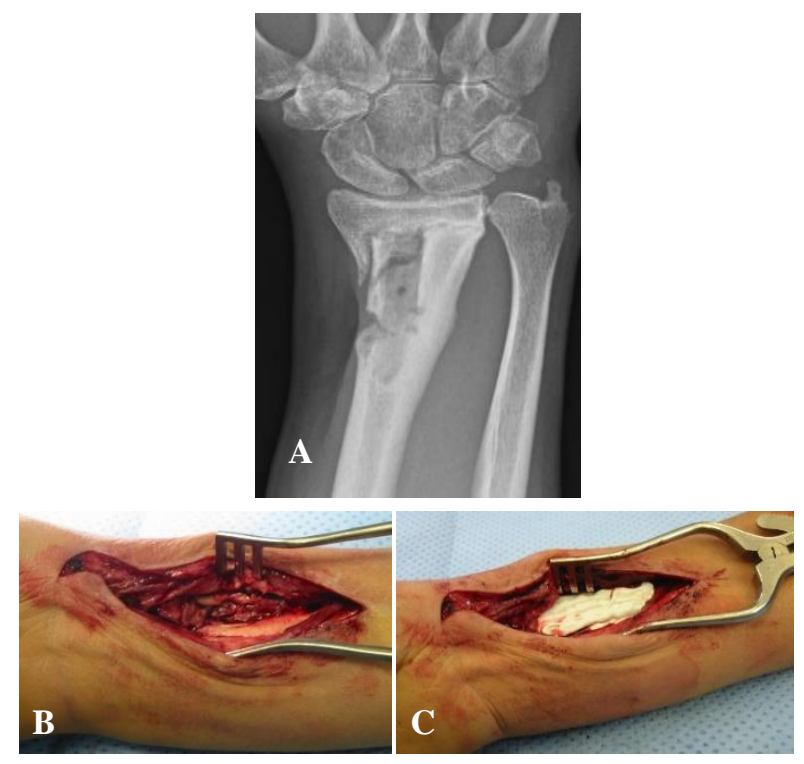

Figure 1: This 30-year-old woman suffered deep infection after internal fixation of a distal radial

fracture $(A)$ the sequestrum and infected tissue was removed $(B)$ the defect completely filled with

Cerament with gentamicin $(C)$ the skin was directly closed over the material.

All patients were commenced on an empiric antibiotic regime of vancomycin and meropenem ${ }^{6}$ at the time of surgery and changed to culture-specific therapy following their operation. No patients were given systemic aminoglycosides in addition to the implanted gentamicin.

Pre-operative serum creatinine levels, along with all creatinine levels measured over the first 7 days following their operation were recorded and used to calculate an estimated glomerular filtration rate (eGFR) using the CKD-epi creatinine equation. ${ }^{22}$ Renal impairment was graded using the CKD staging system. ${ }^{23}$

Outcome measures included clinical signs of kidney dysfunction, acute kidney injury classified according to the RIFLE criteria, and declines in GFR of $>10 \%$ or producing a change in the CKD stage in the first postoperative week. ${ }^{24}$

The RIFLE criteria classify the degree of change in renal function related to the creatinine levels or urine output. A rise in serum creatinine of 1.5 times above baseline defines a potential 'risk' of renal injury. A rise of more than 2 times or 3 times above baseline indicates renal 'injury' or renal 'failure' respectively. ${ }^{24}$ 
It was assumed that data was non-parametric and consequently Mann Whitney U test and Spearman rank correlation were used to test for statistical significance. A $\mathrm{p}<0.05$ was taken to be statistically significant.

\section{RESULTS}

Of the 163 patients identified, 109 were male, with an average age of 51.6 years. Seven patients had pre-existing renal disease identified prior to their operation (4 diabetic nephropathy, 1 nephrotic syndrome, 1 renal transplant and 1 previous AKI).

The anatomic location of the osteomyelitis was in the upper limb in 37 patients, lower limb in 124 and pelvis in 2 (Table 1). The mean volume of antibiotic carrier used was $10.9 \mathrm{ml}$ (range $1-30 \mathrm{ml}$ ), equating to a mean gentamicin dose of $191.3 \mathrm{mg}$. The maximum implanted dose of gentamicin in any patient was $525 \mathrm{mg}$.

Table 1: Anatomic location of osteomyelitis and dose of implanted gentamicin.

\begin{tabular}{|lllll|} 
Gender & $\begin{array}{l}\text { Anatomic } \\
\text { region }\end{array}$ & $\mathbf{N}$ & $\begin{array}{l}\text { Mean } \\
\text { volume } \\
\text { Cerament } \\
\text { G (ml) }\end{array}$ & $\begin{array}{l}\text { Mean } \\
\text { gentamicin } \\
\text { dose (mg) }\end{array}$ \\
\hline Male & Forearm & 5 & 2.6 & 45.5 \\
\hline Male & Humerus & 12 & 7.7 & 134.2 \\
\hline Male & Clavicle & 1 & 1.0 & 17.5 \\
\hline Male & Foot & 4 & 12.0 & 210.0 \\
\hline Male & Ankle/ & 3 & 6.9 & 120.6 \\
\hline Male & Tibula & 59 & 12.8 & 223.9 \\
\hline Male & Femur & 24 & 14.2 & 247.9 \\
\hline Male & Pelvis & 1 & 8.0 & 140.0 \\
\hline Female & Forearm & 8 & 3.0 & 52.5 \\
\hline Female & Humerus & 10 & 7.6 & 132.1 \\
\hline Female & Clavicle & 1 & 3.0 & 52.5 \\
\hline Female & Foot & 4 & 7.8 & 135.6 \\
\hline Female & $\begin{array}{l}\text { Ankle/ } \\
\text { fibula }\end{array}$ & 2 & 6.5 & 113.8 \\
\hline Female & Knee & 1 & 10.0 & 175.0 \\
\hline Female & Tibia & 19 & 11.5 & 200.8 \\
\hline Female & Femur & 8 & 15.0 & 262.5 \\
\hline Female & Pelvis & 1 & 9.0 & 157.5 \\
\hline Total & & 163 & 10.9 & 191.3 \\
\hline & & & & \\
\hline
\end{tabular}

155 patients had both pre-operatives, and at least one post-operative serum creatinine recorded. Patients had an average of three post-operative serum creatinine measurements (range 1-6) in the 7 days following their operation. According to CKD staging prior to their operations, there were $118 \mathrm{CKD}$ class I (normal), 30 class II (mild), 3 class IIIa (moderate), 3 class IIIb (moderate), and 1 class $\mathrm{V}$ (end stage) patients. The mean preoperative serum creatinine was 73.0 (range 39 to 425), equating to a mean eGFR of $98.7 \mathrm{ml} / \mathrm{min} / 1.73 \mathrm{~m}^{2}$ (range 11 to 139$)$.
Seventy patients (45.2\%) developed a fall in eGFR peaking at a mean of 3.06 days after surgery (range 1-7 days; SD 2.133). When the lowest calculated postoperative eGFR was compared to pre-operative levels, the mean greatest decline in eGFR was $-0.92 \mathrm{ml} / \mathrm{min} / 1.73$ $\mathrm{m}^{2}$ (SD 9.07). There was no significant difference in this decline between male $\left(-0.71 \mathrm{ml} / \mathrm{min} / 1.73 \mathrm{~m}^{2}\right)$ and female $\left(-1.33 \mathrm{ml} / \mathrm{min} / 1.73 \mathrm{~m}^{2}\right)$ patients $(\mathrm{p}=0.94)$. Twenty patients $(12.9 \%)$ had a fall in eGFR of $>10 \%$ from baseline, and $16(10.3 \%)$ of these declined by a single CKD stage. Seven (4.5\%) patients fell into the "Risk" category for AKI according to the RIFLE criteria. No patient had a change consistent with "Injury", "Failure" or "Loss" of renal function as classified by the RIFLE criteria. No patient had any clinical signs of new acute renal dysfunction post-operatively. Only one of the seven patients with pre-existing renal disease had an eGFR drop $>10 \%$ (from $11 \mathrm{ml} / \mathrm{min} / 1.73 \mathrm{~m}^{2}$ to $8 \mathrm{ml} / \mathrm{min} / 1.73 \mathrm{~m}^{2}$ ), which did not represent a change in CKD stage.

When the last recorded eGFR in the 7 post-operative days was compared to pre-operative levels, however, the mean change was an increase in eGFR of $4.11 \mathrm{ml} / \mathrm{min} / 1.73 \mathrm{~m}^{2}$ (SD 8.61) above preoperative levels. Only $8(5 \%)$ patients had a persisting reduction in eGFR of $>10 \%$ from baseline at final assessment. Two of these did not have blood tests repeated on days 5 to 7 post-op, so recovery of renal function may potentially have been missed. Five patients remained a single CKD stage worse than pre-operatively and 2 patients remained in the "Risk" category for AKI according to RIFLE criteria at 7 days after surgery.

All patients were followed up clinically to at least one year after surgery (mean 21.4 months; range 12-56). No patient required readmission or treatment for any new renal impairment during the follow-up period.

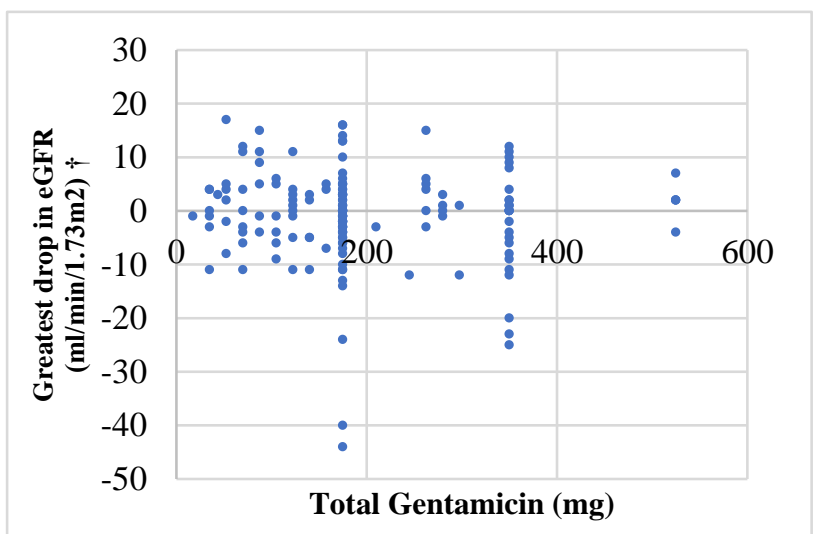

Figure 2: The relationship of implanted gentamicin dose to change in eGFR.

$\uparrow$ a negative value indicates a rise in eGFR after surgery.

No significant correlation was found between the total dose of implanted gentamicin and the greatest decline in post-operative eGFR $\left(\mathrm{r}_{\mathrm{s}}=-0.05963, \mathrm{p}=0.46112\right)$ (Figure 2). Change in eGFR was not related to gentamicin dose. 


\section{DISCUSSION}

Local antibiotic implantation has been established in the treatment of bone and joint infections since at least the 1970 's. ${ }^{25}$ The use of PMMA as a carrier compound has the disadvantage of requiring removal. Additionally, after a short period of high concentration antibiotic release, PMMA continues to elute low levels of antibiotics for many weeks which theoretically confers an increased risk of the development of antibiotic resistance. ${ }^{26,27}$

These concerns have led to increasing interest in biodegradable ceramic antibiotic carriers. ${ }^{15,19,21}$ These carriers dissolve and do not need surgical removal. Their drug elution continues for a limited period of 3-4 weeks before ceasing completely, as the material dissolves and the antibiotic is washed out. ${ }^{14,20}$ The Cerament used in our patients is a biphasic ceramic, consisting of $60 \%$ calcium sulphate and $40 \%$ hydroxyapatite crystals (by weight). The calcium sulphate component acts to deliver the antibiotic, while the hydroxyapatite persists and is gradually remodelled to living bone over several years. ${ }^{28}$

Gentamicin is the most widely used antibiotic for local delivery in osteomyelitis treatment. It is a bactericidal agent, and is active against gram negative organisms and gram-positive organisms in higher concentrations. Natural bacterial resistance to gentamicin is rare and acquired resistance remains uncommon. Systemic gentamicin therapy is well recognised to carry the risk of two major side effects, nephrotoxicity and ototoxicity. ${ }^{16,17,29}$ The toxic effect of aminoglycosides on renal function is caused mainly by effects on proximal tubular epithelial cells in the renal cortex. The drug is actively transported into these cells across the cell membrane and concentrates in endosomal structures including lysosomes, Golgi and the endoplasmic reticulum. This interferes with cell function and, when sufficiently high concentrations are reached, disrupts the endosome membrane, setting off a chain of events that culminates in cell death and acute tubular necrosis. Gentamicin also produces inflammatory change in the glomerular basement membrane, leading to mesangial cell contraction and loss of selectivity in the glomerular filtration barrier. These combined effects lead to a fall in glomerular filtration rate, loss of electrolyte homeostasis and proteinuria.

Nephrotoxicity was reported in $10-25 \%$ of patients in earlier series, where multiple daily systemic dosing regimens were used. ${ }^{29,30}$ More recently it has been recognised that once daily dosing, producing high peak and low trough levels of the drug in serum, maintains its antibacterial efficacy while reducing toxic renal effects. ${ }^{31}$ In contradiction to this systemic dosing pattern, the release from local antibiotics carriers, such as the Cerament used in this study, occurs at a fairly constant rate, with fluctuations in local and serum gentamicin levels occurring over days to weeks rather than within any 24-hour period. ${ }^{14}$ Our results suggest that this does not appear to produce a similar level of renal insult to systemic use. The observed changes in eGFR had no relationship to the implanted gentamicin dose (Figure 2).

A transient increase in serum creatinine (and consequent decrease in eGFR) was seen in less than half of our patients, with the greatest change occurring at around 3 days following surgery. The scale of the fall in eGFR was, however, small, with most being within the range of normal daily variation within individuals. Of the 120 patients with a pre-operative eGFR $>90 \mathrm{ml} / \mathrm{min} / 1.73 \mathrm{~m}^{2}$ (CKD class I, normal renal function), 110 remained within this normal range throughout the study period. No patient in our series developed a clinically significant kidney injury. Although 20/155 (12.9\%) experienced a fall in eGFR of at least $10 \%$, more than half of these $(12 / 20,60 \%)$ resolved within 7 days from the operation. There was no evidence of declining renal function over the seven postoperative days due to any potential accumulation of gentamicin toxicity.

The RIFLE criteria are a validated, internationally agreed tool for the diagnosis of AKI. ${ }^{24}$ It has been demonstrated to be predictive of the need for renal replacement therapy and mortality in hospitalised patients. ${ }^{32-34}$ of the 7 patients in our study whose fall in eGFR put them in the "Risk" category of the RIFLE criteria, in five the decline in renal function resolved within the 7 days following the operation. Of the remaining two, one had end-stage $(\mathrm{CKD}$ Class V) renal failure prior to their operation secondary to diabetic nephropathy. The eGFR in this patient fell from 11 to $8 \mathrm{ml} / \mathrm{min} / 1.73 \mathrm{~m}^{2}$ which had no clinical significance in terms of their treatment. The second patient was a 68-year-old male with peripheral vascular disease, who was taking ramipril for hypertension. On the fourth post-operative day he developed an intestinal pseudo-obstruction that was decompressed by flexible sigmoidoscopy two days later. It was during this period that the decline in his renal function occurred, and it is likely that the large fluid shifts associated with the bowel obstruction contributed to his fall in eGFR. This last patient serves as a reminder that an acute kidney injury is often multifactorial and that a decline in renal function is not uncommonly seen following major surgical procedures, even in the absence of aminoglycoside antibiotics. In their series of over 12,000 patient undergoing surgery in Scottish NHS hospitals, Bell et al demonstrated an incidence of $6.2 \%$ post-operative AKI in patients having orthopaedic implant procedures when aminoglycosides were not used for surgical prophylaxis..$^{35}$

The incidence of AKI rose to $10.8 \%$ when a single intravenous dose of gentamicin ( $4 \mathrm{mg} / \mathrm{kg}$ ) was used. Changes in organ perfusion and fluid balance during anaesthesia and other systemic drugs, can have a profound impact on renal function. Our incidence of $4.5 \%$ of patients falling into the 'Risk' category for AKI represents a very low rate of mild post-operative renal dysfunction. 
None of our patients received systemic aminoglycoside antibiotics in addition to the implanted Cerament G, but all received intravenous vancomycin as empiric treatment prior to culture and sensitivity results being available. Although less nephrotoxic than aminoglycosides, vancomycin can be harmful to renal function, albeit to a mild and reversible degree in most cases. ${ }^{36}$ Vancomycin has also been shown to increase the risk of AKI in patients receiving systemic gentamicin, and can produce rates of $\mathrm{AKI}$ in excess of $17 \%$ when given to treat osteomyelitis in combination with other antibiotics. ${ }^{37,38}$ This synergistic effect was not observed in our patients, who received only locally delivered gentamicin.

One limitation of this study is the lack of a control group of patients who had the same surgery without Cerament $\mathrm{G}$ implantation. We are therefore unable to comment on the background rate of AKI with this surgery, in the absence of local gentamicin. Our results are specific to this antibiotic carrier and should not be extended to infer any safety profile for any other biodegradable delivery material.

Serum creatinine and calculated eGFR were used as practical and measurable surrogates of renal function. The measured value of serum creatinine depends on the relationship between its production in skeletal muscle, and its clearance in the kidneys. In health, the production is consistent, but sepsis and critical illness may cause a fall in production. ${ }^{39.40}$ This may explain the rise in mean eGFR in our patient group after treatment of the osteomyelitis. The concept of renal reserve also means that the relationship between creatinine and GFR is nonlinear, so the same absolute change in creatinine will have differing significance depending on baseline renal function.

\section{CONCLUSION}

The implantation of up to $525 \mathrm{mg}$ of gentamicin contained within Cerament $G$, as part of the surgical treatment of osteomyelitis, is safe and carries minimal risk of significant acute kidney injury. A small, transient increase in serum creatinine may be observed in the early post-operative period, and attention should be paid to limit patients' exposure to other nephrotoxic agents. The majority of patients will return to their baseline renal function within 7 days following the operation. Specific treatment was not required in any of our patients.

\section{ACKNOWLEDGEMENTS}

The authors would like to thank the patients and staff of the Oxford bone infection unit for their support in this study.

\section{Funding: No funding sources}

Conflict of interest: None declared

Ethical approval: The study was approved by the institutional ethics committee

\section{REFERENCES}

1. McNally M, Nagarajah K. Osteomyelitis. Orthop Trauma. 2010;24(6):416-29.

2. Metsemakers W-J, Morgenstern, M, Senneville E. General treatment principles for fracture-related infection: recommendations from an international expert group. Arch Orthop Trauma Surg. 2020;140:1013-27.

3. Chan JKK, Ferguson JY, Scarborough M, McNally MA, Ramsden AJ. Management of Post-Traumatic Osteomyelitis in the Lower Limb: Current State of the Art. Indian J Plast Surg. 2019;52(1):62-72.

4. Cierny III G, DiPasquale D. Treatment of chronic infection. J Am Acad Orthop Surg. 2006;14:S10510.

5. BMJ Best Practice Guideline for osteomyelitis. Available https://bestpractice.bmj.com/topics/en-gb/354.

Accessed 8 November, 2020.

6. Dudareva M, Hotchen AJ, Ferguson J, Hodgson S, Scarborough M, Atkins BL et al. The microbiology of osteomyelitis: changes over ten years. J Infection. 2019;79:189-98.

7. Li HK, Rombach I, Zambellas R. Oral versus Intravenous Antibiotics for Bone and Joint Infection. N Engl J Med. 2019;380(5):425-36.

8. Kruse Jensen L, Koch J, Hendriksen L, Bue M, Tottrup M, Hanberg $\mathrm{P}$ et al. Suppurative Inflammation and Local Tissue Destruction Reduce the Penetration of Cefuroxime to Infected Bone Implant Cavities. J Comparative Pathol. 2017;157(4):308-16.

9. Jensen LK, Bjarnsholt T, Kragh KN, Aalbæk B, Henriksen NL, Blirup SA et al. In vivo gentamicin susceptibility test for prevention of bacterial biofilms in bone tissue and on implants. Antimicrob Agents Chemother. 2019;63:e0188.

10. Rand BC, Penn-Barwell JG, Wenke JC. Combined local and systemic antibiotic. delivery improves eradication of wound contamination: an animal experimental model of contaminated fracture. Bone Joint J. 2015;97-B:1423-7.

11. Ruppen C, Hemphill A, Sendi P. In-vitro activity of gentamicin as adjunct to penicillin against biofilm group B Streptococcus. J Antimicrob Chemother. 2017;72:444-7.

12. Wahlig H, Dingeldein E. Antibiotics and bone cements. Experimental and clinical long-term observations. Acta Orthop Scand. 1980;52(1):49-56.

13. Moojen DJ, Hentenaar B, Vogely $\mathrm{CH}$. In vitro release of antibiotics from commercial PMMA beads and articulating hip spacers. J Arthroplasty. 2008;23:1152-6.

14. Stravinskas M, Horstmann P, Ferguson J, Hettwer W, Nilsson M, Tarasevicius $S$ et al. Pharmacokinetics of gentamicin eluted from a regenerating bone graft substitute - In vitro and clinical release studies. Bone Joint Res. 2016;5:42735 
15. Ferguson J, Diefenbeck M, McNally M. Ceramic bio composites as biodegradable antibiotic carriers in the treatment of bone infections. J Bone Joint Infect. 2017;2:41-54.

16. Selby NM, Shaw S, Woodier N, Fluck RJ, Kolhe NV. Gentamicin-associated acute kidney injury. QJM. 2009;102(12):873-80.

17. Hayward RS, Harding J, Molloy R, Land L, Longcroft-Neal K, Moore D, Ross JDC. Adverse effects of a single dose of gentamicin in adults: a systematic review. $\mathrm{Br} \mathrm{J}$ Clin Pharmacol. 2018;84:223-38.

18. Walenkamp GH, Vree TB, van Rens TJ. GentamicinPMMA beads. Pharmacokinetic and nephrotoxicological study. Clin Orthop Relat Res. 1986;205:171-83.

19. Metsemakers W-J, Fragomen AT, Moriarty F, Morgenstern M, Egol KA, Zalavras $\mathrm{C}$ et al. Evidence-based recommendations for local antimicrobial strategies and dead space management in Fracture-related Infection. J Orthop Trauma. 2019;34:18-29.

20. Oliver RA, Lovric V, Christou C, Walsh WR. Evaluation of comparative soft tissue response to bone void fillers with antibiotics in a rabbit intramuscular model. J Biomaterials Applications. 2019;34(1):117-29.

21. McNally M, Ferguson J, Diefenbeck M. Single-stage treatment of chronic osteomyelitis with a new absorbable, gentamicin-loaded, calcium sulphate/hydroxyapatite bio-composite. A prospective series of 100 cases. Bone Joint J. 2016;98-B:1289-96.

22. Levey AS, Stevens LA, Schmid CH. A new equation to estimate glomerular filtration rate. Ann Intern Med. 2009;150(9):604-12.

23. K/DOQI clinical practice guidelines for chronic kidney disease: Evaluation, classification, and stratification. Am J Kidney Dis. 2002;39:S1-266.

24. Bellomo R, Ronco C, Kellum JA. Acute renal failure-definition, outcome measures, animal models, fluid therapy and information technology needs: the Second International Consensus Conference of the Acute Dialysis Quality Initiative (ADQI) Group. Crit Care. 2004;8:R204.

25. Klemm K. The treatment of chronic bone infections with gentamicin-PMMA-chains and beads. $\mathrm{H}$ Contzen (Ed.), Gentamycin-PMMA-Kette, Unfallchirurgie. 1977;3(1):20-5.

26. Neut D, van de Belt $H$, van Horn JR, van der Mei $\mathrm{HC}$, Busscher HJ. Residual gentamicin-release from antibiotic-loaded polymethylmethacrylate beads after 5 years of implantation. Biomaterials. 2003;24(10):1829-31.

27. Thomes B, Murray P, Bouchier-Hayes D. Development of resistant strains of Staphylococcus epidermidis on gentamicin-loaded bone cement in vivo. J Bone Joint Surg Br. 2002;84:758-60.
28. Ferguson J, Athanasou N, Diefenbeck M, McNally M. Radiographic and Histological Analysis of a Synthetic Bone Graft Substitute Eluting Gentamicin in the Treatment of Chronic Osteomyelitis. J Bone Jt Infect. 2019;4(2):76-84.

29. Smith CR, Lipsky JJ, Laskin OL, Hellmann DB, Mellits ED, Longstreth $\mathrm{J}$ et al. Double-blind comparison of the nephrotoxicity and auditory toxicity of gentamicin and tobramycin. $\mathrm{N}$ Engl $\mathrm{J}$ Med. 1980;302:1106-9.

30. Matzke GR, Lucarotti RL, Shapiro HS. Controlled comparison of gentamicin and tobramycin nephrotoxicity. Am J Nephrol. 1983;3(1):11-7.

31. Nicolau DP, Freeman CD, Belliveau PP, Nightingale $\mathrm{CH}$, Ross JW, Quintiliani R. Experience with a oncedaily aminoglycoside program administered to 2,184 adult patients. Antimicrob Agents Chemother. 1995;39(3):650-55.

32. Uchino S, Bellomo R, Goldsmith D. An assessment of the RIFLE criteria for acute renal failure in hospitalized patients. Crit Care Med. 2006;34:19137.

33. Ostermann M, Chang RW. Acute kidney injury in the intensive care unit according to RIFLE. Crit Care Med. 2007;35:1837-43.

34. Hoste EA, Clermont G, Kersten A. RIFLE criteria for acute kidney injury are associated with hospital mortality in critically ill patients: a cohort analysis. Crit Care. 2006;10:R73.

35. Bell S, Davey $P$, Nathwani D, Marwick C, Vadiveloo T, Sneddon J et al. Risk of AKI with Gentamicin as Surgical Prophylaxis. JASN. 2014;25(11):2625-32.

36. Mergenhagen KA, Borton AR. Vancomycin nephrotoxicity: a review. J Pharm Pract. 2014;27(6):545-53.

37. Rybak MJ, Abate BJ, Kang SL, Ruffing MJ, Lerner SA, Drusano GL. Prospective evaluation of the effect of an aminoglycoside dosing regimen on rates of observed nephrotoxicity and ototoxicity. Antimicrob Agents Chemother. 1999;43(7):1549-55.

38. Moenster RP, Linneman TW, Finnegan PM, Hand S, Thomas Z, McDonald JR. Acute renal failure associated with vancomycin and $\beta$-lactams for the treatment of osteomyelitis in diabetics: piperacillintazobactam as compared with cefepime. Clin Microbiol Infect. 2014;20(6):O384-9.

39. Doi K, Yuen PS, Eisner C, Hu X, Leelahavanichkul A, Schnermann $J$ et al Reduced production of creatinine limits its use as marker of kidney injury in sepsis. J Am Soc Nephrol. 2009;20(6):1217-21.

40. Clark WR, Mueller BT, Kraus MA, Macias WL. Quantification of creatinine kinetic parameters in patients with acute renal failure. Kidney Int. 1998;54(2):554-60.

Cite this article as: Muir R, Birnie $\mathrm{C}$, HyderWilson R, Ferguson J, McNally MA. Does local implantation of gentamicin impair renal function in patients undergoing surgery for chronic bone infection? Int J Res Orthop 2021;7:438-43. 